\title{
WEYL'S THEOREM THROUGH LOCAL SPECTRAL THEORY*
}

\author{
SLAVIŠA DJORDJEVIĆ \\ Faculty of Science, Department of Mathematics, University of Niš, \\ Cirila i Metodija 2, 18000 Niš, Yugoslavia \\ e-mail:slavdj@pmf.pmf.ni.ac.yu \\ IN HO JEON and EUNGIL KO \\ Department of Mathematics, Ewha Women's University, Seoul 120-750, Korea \\ e-mail:jih@math.ewha.ac.kr,eiko@mm.ewha.ac.kr
}

(Received 18 September, 2000; accepted 16 March 2001)

\begin{abstract}
In this paper, we show that Weyl's theorem holds for operators having the single valued extension property and quasisimilarity preserves Weyl's theorem for these operators under some assumptions for spectral subsets, respectively.
\end{abstract}

2000 Mathematics Subject Classification. 47A53, 47A10.

1. Introduction. Let $\mathcal{X}$ be an infinite dimensional complex Banach space and let $\mathcal{L}(\mathcal{X})$ be the space of all bounded linear operators acting on a Banach space $\mathcal{X}$. For an operator $T \in \mathcal{L}(\mathcal{X})$, we shall denote by $\sigma(T), \sigma_{p}(T), \pi_{0}(T)$ and iso $\sigma(T)$ the spectrum of $T$, the set of all eigenvalues of $T$, the set of all eigenvalues of finite multiplicity of $T$, the set of all isolated points of $\sigma(T)$, respectively. We write $\pi_{00}(T)=$ $\pi_{0} \cap$ iso $\sigma(T)$ for the set of all isolated eigenvalues of finite multiplicity of $T$. Recall ([5], [7]) that an operator $T \in \mathcal{L}(\mathcal{X})$ is called semi-Fredholm if the range of $T$, denoted by $R(T)$, is closed and either the kernel of $T$, denoted by $N(T)$, or $X / R(T)$ is finite dimensional. Also, an operator $T \in \mathcal{L}(\mathcal{X})$ is called Fredholm if $R(T)$ is closed and both $N(T)$ and $X / R(T)$ are finite dimensional. If $T$ is semi-Fredholm, then the index of $T$ is defined by $\operatorname{ind}(T)=\operatorname{dim} N(T)-\operatorname{dim} X / R(T)$ and a Fredholm operator with index zero is called Weyl. Also, if $T$ is Fredholm and there exists a deleted open neighborhood $N_{0}$ of $0 \in \mathbb{C}$ such that $T-\lambda$ is invertible for all $\lambda \in N_{0}$, then $T$ is said to be Browder. We shall denote by $\sigma_{s F}(T), \sigma_{e}(T), \omega(T)$, and $\sigma_{b}(T)$ the semi-Fredholm spectrum, the (Fredholm) essential spectrum, the Weyl spectrum, and the Browder spectrum of $T$, respectively. We easily see the following inclusions:

$$
\sigma_{s F}(T) \subseteq \sigma_{e}(T) \subseteq \omega(T) \subseteq \sigma_{b}(T)
$$

We usually say that Weyl's theorem holds for $T$ if $\sigma(T) \backslash \omega(T)=\pi_{00}(T)$. In [2] Coburn showed that Weyl's theorem holds for hyponormal operators and Toeplitz operators acting on a Hilbert space. After Coburn's work, many authors have extended Weyl's theorem to some classes of operators on a Hilbert space (see [1], [13], etc.) or on a Banach space (e.g., [6], [9], [14], etc.). Throughout this paper we shall mainly study Weyl's theorem for operators on a Banach space.

*This work of the second and the third authors was supported by the Brain Korea 21 project. 
Regarding local spectral theory ([3], [10]) recall that if $T \in \mathcal{L}(\mathcal{X})$ and $F$ is a closed subset of the complex plane $\mathbb{C}$, we define a spectral manifolds $\mathcal{X}_{T}(F)$ as follows:

$$
\begin{array}{r}
\mathcal{X}_{T}(F)=\{x \in \mathcal{X}: \text { there exists an analytic } \mathcal{X} \text {-valued function } \\
f: \mathbb{C} \backslash F \longrightarrow \mathcal{X} \text { such that }(T-\lambda) f(\lambda)=x\} .
\end{array}
$$

We say that $T$ has the single valued extension property (SVEP) at $\lambda_{\circ} \in \mathbb{C}$ if, for a neighborhood $U$ of $\lambda_{\circ}, f=0$ is the only analytic function $f: U \rightarrow \mathcal{X}$ satisfying $(T-\lambda) f(\lambda)=0$. Also, we say that $T$ has the SVEP if $T$ has this property at every $\lambda \in \mathbb{C}$. In this case, for $x \in \mathcal{X}$ there is a maximal analytic function $f_{x}: U_{x} \rightarrow \mathcal{X}$ satisfying $(T-\lambda) f_{x}(\lambda)=x$ on $U_{x}$. Put $\sigma_{T}(x)=\mathbb{C} \backslash U_{x}$. Then $\sigma_{T}(x)$ is called the local spectrum at $x$. If $T$ has the SVEP, then we have $\mathcal{X}_{T}(F)=\left\{x \in \mathcal{X}: \sigma_{T}(x) \subseteq F\right\}$. We say that $T$ has Dunford's property $(C)$ if, for each closed set $F \subset \mathbb{C}, \mathcal{X}_{T}(F)$ is closed. Also, we say that $T$ has Bishop's property $(\beta)$ if for every sequences $f_{n}: U \rightarrow \mathcal{X}$ such that $(T-\lambda) f_{n} \rightarrow 0$ uniformly on compact subsets in $U$, it follows that $f_{n} \rightarrow 0$ uniformly on compact subsets in $U$. Then it is well known that

$$
\text { Bishop's property }(\beta) \Rightarrow \text { Dunford's property }(C) \Rightarrow \text { SVEP. }
$$

We consider a condition given in terms of spectral manifolds of $T \in \mathcal{L}(\mathcal{X})$ :

$$
\mathcal{X}_{T}(\{\lambda\}) \text { is finite dimensional for all } \lambda \in \pi_{00}(T) \text {. }
$$

For brevity, we shall denote the classes of all operators having the single valued extension property, having the condition (4), and having Dunford's property (C) by $A, \mathcal{B}$, and $\mathcal{D}$, respectively.

Recall ([9], [10], [11], [15], [16]) that $T, S \in \mathcal{L}(\mathcal{X})$ are said to be quasisimilar if there exist injections $X, Y \in \mathcal{L}(\mathcal{X})$ with dense range such that $S X=X T$ and $Y S=T Y$, respectively, and this relation of $T$ and $S$ is denoted by $T \sim S$.

The aim of this paper is to consider Weyl's theorem from the standpoint of local spectral theory and quasisimilarity. In particular, we show that Weyl's theorem holds for operators in class $\mathcal{A} \cap \mathcal{B}$ and that quasisimilarity preserve Weyl's theorem for some operators in class $\mathcal{A}$. This extends some results proved in [9].

2. Main results. We consider Weyl's theorem through local spectral theory. To do so, we need the following two results that play the role of a bridge between Fredholm and local spectral theory. The first proposition is due to K. B. Laursen.

Proposition A. ([11, Lemma 1]) If $\lambda \in$ iso $\sigma(T)$, then $\lambda \notin \sigma_{s F}(T)$ if and only if $\lambda \notin \sigma_{e}(T)$ if and only if $\mathcal{X}_{T}(\{\lambda\})$ is finite dimensional.

Recall ([7]) that the ascent $p$ of $T \in \mathcal{L}(\mathcal{X})$ at $\lambda \in \mathbb{C}$ is the extended integer given by $p=\inf \left\{n \geq 0: N(T-\lambda)^{n}=N(T-\lambda)^{n+1}\right\}$. The infimum over the empty set is taken to be $\infty$. The following one is due to J. K. Finch.

Proposition B. ([4, Theorem 15]) Let $T \in \mathcal{L}(\mathcal{X})$ be semi-Fredholm. Then $T$ has the single valued extension property at 0 if and only if $T$ has a finite ascent at 0.

Then we can observe the following fact. 
Lemma I. For every $\lambda \in \pi_{00}(T), \mathcal{X}_{T}(\{\lambda\})$ is finite dimensional if and only if $R(T-\lambda)$ is closed.

Proof. Assume that $\mathcal{X}_{T}(\{\lambda\})$ is finite dimensional for $\lambda \in \pi_{00}(T)$. Then, by Proposition A, $T-\lambda$ is semi-Fredholm, and so $R(T-\lambda)$ is closed. Conversely, assume that $R(T-\lambda)$ is closed for $\lambda \in \pi_{00}(T)$. Then $T-\lambda$ is (upper) semi-Fredholm since $\operatorname{dim} N(T-\lambda)<\infty$. Thus, by Proposition A again, we have that $\mathcal{X}_{T}(\{\lambda\})$ is finite dimensional.

Now, we have the following result.

Theorem 2. If $T \in \mathcal{A} \cap \mathcal{B}$, then Weyl's theorem holds for $T$.

Proof. If $\lambda \in \sigma(T)-\omega(T)$, then $T-\lambda$ is Weyl but not invertible. Since $T \in \mathcal{A}$ and the SVEP has the translation invariant property for scalars, by Proposition $\mathrm{B}$ we have that the ascent of $T$ is finite. Thus, by [5, Theorem 7.9.3], $T-\lambda$ is Browder, and so $\lambda \in$ iso $\sigma(T)$. Hence we have $\lambda \in \pi_{00}(T)$ because $\sigma(T)-\omega(T) \subset \pi_{0}(T)$.

Conversely, assume that $\lambda \in \pi_{00}(T)$, i.e. $\lambda \in$ iso $\sigma(T)$ and $\operatorname{dim} N(T-\lambda)<\infty$. Then $T-\lambda$ is a semi-Fredholm operator since $T \in \mathcal{B}$, and so by Lemma 1 and Proposition A $T-\lambda$ is Fredholm. Furthermore, $T-\lambda$ is Browder because $\lambda \in$ iso $\sigma(T)$. Thus $T-\lambda$ is Weyl, and hence $\pi_{00}(T) \subset \sigma(T)-\omega(T)$.

Recall [9] that an operator $T \in \mathcal{L}(\mathcal{X})$ is said to be finitely ascensive if for every $\lambda \in \mathbb{C}$ there is a $p \in \mathbb{N}$ such that $N(T-\lambda)^{p}=N(T-\lambda)^{p+1}$. It is well known ([12, Proposition 1.8]) that if $T$ is finitely ascensive, then it has SVEP. Thus Theorem 2 above is an extension of Theorem 2 in [9] in view of Lemma 1.

The class $\mathcal{B}$ may seem artificial, and so we consider a more concrete class of operators contained in $\mathcal{B}$. Recall ([17]) that $T \in \mathcal{L}(\mathcal{X})$ is said to have a growth condition $G_{m}(m \geq 1)$ if there exists a constant $K$ such that

$$
\left\|(T-\lambda)^{-1}\right\| \leq \frac{K}{\operatorname{dist}(\lambda, \sigma(T))^{m}}, \text { for all } \lambda \notin \sigma(T) .
$$

We shall denote by $\mathcal{G}_{m}$ the set of all operators with the growth condition $G_{m}(m \geq 1)$. In the context of Hilbert space many properties of operators in $\mathcal{G}_{m}$ have been well studied in [17], and especially, when $m=1$, in [8].

Using an argument similar to that of Theorem 14 of [6], we have the following result.

\section{Lemma 3. $\mathcal{G}_{m} \subset \mathcal{B}$.}

Proof. Assume $T \in \mathcal{G}_{m}$ and $\lambda \in \pi_{00}(T)$. If we consider the Riesz projection $P_{\lambda}^{T}$ corresponding to $\lambda$ for $T$, then $\left.(T-\lambda)\right|_{R\left(P_{\lambda}^{T}\right)}$ is quasi-nilpotent. Thus, by [7, Proposition 49.1], we see that

$$
R\left(P_{\lambda}^{T}\right)=\left\{x \in \mathcal{X}:\left\|(T-\lambda)^{n} x\right\|^{\frac{1}{n}} \rightarrow 0\right\}=\mathcal{X}_{T}(\{\lambda\}) .
$$

Now, since $T \in \mathcal{G}_{m}$, letting $0<\epsilon \leq \frac{1}{K} \operatorname{dist}(\lambda, \sigma(T))^{m}$, we can see that 


$$
\|\left.(T-\lambda)\right|_{R\left(P_{\lambda}^{T}\right)}|| \leq \frac{1}{2 \pi} \int_{|z|=\epsilon}|z|||\{(T-\lambda)-z\}^{-1}|||d z| \leq \epsilon,
$$

where the circular contour is described once counterclockwise. Thus $\left.(T-\lambda)\right|_{R\left(P_{\lambda}^{T}\right)}$ is a zero operator. So $R\left(P_{\lambda}^{T}\right) \subseteq N(T-\lambda)$ and the reverse containment is easily verified, and consequently we have the equality $R\left(P_{\lambda}^{T}\right)=N(T-\lambda)$. Since $N(T-\lambda)$ is finite dimensional, by the assumption we get that $\mathcal{X}_{T}(\{\lambda\})$ is finite dimensional by (5). Hence $T \in \mathcal{B}$.

\section{COROLlary 4. Weyl's theorem holds for $T \in \mathcal{A} \cap \mathcal{G}_{m}$.}

It is well known that the class of spectral operators of finite type is contained in $\mathcal{A} \cap \mathcal{G}_{m}$ by Theorem XVI.4.4 and Theorem XV.6.7 of [3]. Thus by Corollary 3 Weyl's theorem holds for a spectral operator of finite type. Hence we can recapture Theorem 4 of Oberai ([14]).

Now, we study Weyl's theorem under quasisimilarity. M. Putinar ([15]) showed that if $T$ and $S$ have Bishop's property $(\beta)$ and $T \sim S$, then quasisimilarity of $T$ and $S$ preserves spectra, essential spectra, and indices of $T$ and $S$. From this fine theorem we immediately observe the following interesting result.

Proposition C. Let $T, S \in \mathcal{L}(\mathcal{X})$ have Bishop's property $(\beta)$. If $T \sim S$, then Weyl's theorem holds for $T$ if and only if Weyl's theorem holds for $S$.

We give an improvement of Proposition $\mathrm{C}$ and Corollary 8 in [9] as follows.

Theorem 5. Let $T, S \in \mathcal{A}$ and let iso $\sigma(T)=$ iso $\sigma(S)$. If $T \sim S$, then Weyl's theorem holds for $T$ if and only if Weyl's theorem holds for $S$.

Proof. Assume Weyl's theorem holds for $T$ and $\lambda \in \pi_{00}(S)$. Since $T \sim S$ it is easy to see that $\pi_{0}(T)=\pi_{0}(S)$. Since iso $\sigma(T)=$ iso $\sigma(S)$ by hypothesis, we have $\pi_{00}(T)=\pi_{00}(S)$. Since Weyl's theorem holds for $T, \lambda \in \sigma(T) \backslash \omega(T)=\pi_{00}(T)$, and so $T-\lambda$ is Weyl. Thus $\mathcal{X}_{T}(\{\lambda\})$ is finite dimensional by Proposition A. Since $T \sim S$ by assumption, there exists an injection $Y \in \mathcal{L}(\mathcal{X})$ with dense range such that $Y S=T Y$, and so we see that

$$
Y \mathcal{X}_{S}(\{\lambda\}) \subseteq \mathcal{X}_{T}(\{\lambda\})
$$

Indeed, it immediately follows that for $x \in \mathcal{X}_{S}(\{\lambda\})$

$$
\left\|(T-\lambda)^{n} Y x\right\|^{1 / n}=\left\|Y(S-\lambda)^{n} x\right\|^{1 / n} \leq\|Y\|^{1 / n}\left\|(S-\lambda)^{n} x\right\|^{1 / n} \longrightarrow 0,
$$

and so from (6), $\mathcal{X}_{S}(\{\lambda\})$ is finite dimensional for $\lambda \in \pi_{00}(S)$. Hence we have $S \in \mathcal{B}$. Since $S$ is already in $\mathcal{A}$ we conclude that Weyl's theorem holds for $S$ by Theorem 2 . Conversely, similar arguments can be applied to complete the proof.

Let $T, S \in \mathcal{L}(\mathcal{X})$ have Bishop's property $(\beta)$ and let $T \sim S$. Then we see that $T, S \in \mathcal{A}$ from (3) and, since $T$ and $S$ have equal spectra from [15, Theorem 1], we have iso $\sigma(T)=$ iso $\sigma(S)$. Consequently, we recapture Proposition $\mathrm{C}$ by Theorem 5 . 
We conclude this paper with a more concrete improvement of Proposition $\mathrm{C}$ in a Hilbert space $\mathcal{H}$.

Corollary 6. Let $T, S \in \mathcal{L}(\mathcal{H})$ have Dunford's property (C). If $T \sim S$, then Weyl's theorem holds for $T$ if and only if Weyl's theorem holds for $S$.

Proof. In [16], J. G. Stampfli showed that quasi-similar operators which satisfy Dunford's property (C) have equal spectra. Thus the proof immediately follows from Theorem 5.

\section{REFERENCES}

1. M. Cho, M. Itoh, and S. Oshiro, Weyl's theorem holds for p-hyponormal operators, Glasgow Math. J. 39 (1997), 217-220.

2. L. A. Coburn, Weyl's theorem for nonnormal operators, Michigan Math. J. 13 (1966), 285-288. 1971).

3. N. Dunford and J. T. Schwartz, Linear operators, part III; spectral operators (Wiley,

4. J. K. Finch, The single valued extension property on a Banach space, Pacific J. Math. 58 (1975), 61-69. 1988).

5. R. E. Harte, Invertibility and singularity for bounded linear operators (Marcel Dekker,

6. R. E. Harte and W. Y. Lee, Another note on Weyl's theorem, Trans. Amer. Math. Soc. 349 (1997), 2115-2124

7. H. G. Heuser, Functional analysis (John Wiley \& Sons, 1982).

8. V. I. Istratescu, Introduction to linear operator theory (Marcel Dekker, 1981).

9. I. H. Jeon, Weyl's theorem and quasisimilarity, Int. Eq. Op. Th. 39 (2001), 214-221. 1992).

10. R. Lange and S. Wang, New approaches in spectral decomposition (Amer. Math Soc.,

11. K. B. Laursen, Essential spectra through local spectral theory, Proc. Amer. Math. Soc. 125 (1997), 1425-1434.

12. K. B. Laursen, Operators with finite ascent, Pacific J. Math. 157 (1992), 323-336

13. W. Y. Lee and S. H. Lee, A spectral mapping theorem for the Weyl spectrum, Glasgow Math. J. 38 (1996), 61-64.

14. K. K. Oberai, On the Weyl spectrum, Illinois J. Math. 18 (1974), 208-212.

15. M. Putinar, Quasi-similarity of tuples with Bishop's property ( $\beta)$, Int. Eq. Op. Th. 15 (1992), 1047-1052. $109-119$

16. J. G. Stampfli, Quasisimilarity of operators, Proc. Roy. Irish Acad Sect. A 81 (1981),

17. B. L. Wadhwa, Spectral, M-hyponormal and decomposable operators, Ph.D. thesis (Indiana University, 1971). 